\title{
THE AUTONOMOUS REPUBLIC OF ADJARA (BASED ON THE EXAMPLE OF GODERDZI SKI RESORT)
}

\author{
PAATA BERIDZE \\ PhD student \\ Batumi Shota Rustaveli State University, Georgia. \\ paataberidze@yahoo.com
}

\begin{abstract}
In the Autonomous Republic of Adjara, the establishment of ski resort on Goderdzi Pass is important for the development of recreation resources and as well as for the central government, which dedicates a significant amount of financial resources from both local and the central budget. Consequently, it is important to consider the ongoing processes of resort privatization. This interest is further intensified by the direct sale of a large amount of real estate. This article analyses the assessment aspects of real estate transfer in the form of direct sales and discusses the factors hindering the development of the resort, as well as the expected consequences of massive real estate privatization. So far, the mechanisms of privatization of property owned by the Autonomous Republic of Adjara and the legislation in force within this area, including strengths and weaknesses are discussed. Based on the law, the goal of privatization in the form of direct sale is to transfer the property right to the customer, who fully and conscientiously fulfils the condition(s) set for property privatization in the form of direct sale. The following can be assessed by the projects and activities carried out by the bidder in the past. The examples of direct selling discussed in the article clearly show that there are frequent cases when the transfer of property in the form of direct sale takes place to entrepreneurial entities registered a few days earlier, which excludes from consideration their experience in this particular field. Apart from that, in the legislative part of maintaining the profile as well as in the part of the employment of the locals, different deadlines and terms of condition are set for various companies which differ greatly without any regularity. Due to the abovementioned reasons, the investors who initially agree with the contract initiate amendments which causes changes to be made to the initial agreement. Often, these changes contradict the general plan for the development of the Goderdzi Ski Resort and have an overall negative impact on the stance of the Autonomous Republic of Adjara due to frequent extension of the deadline for the investment execution. Based on the results of the research, it is recommended to create a document (computing formula) that will allow the property management body to determine the minimum amount of employees and the amount of investment during the development of the investment package, as well as the minimum period of profile maintenance.
\end{abstract}

KEYWORDS: PROPERTY OF THE AUTONOMOUS REPUBLIC OF AJARA; DIRECT SELLING; RESORT GODERDZI; LAW OF THE AUTONOMOUS REPUBLIC OF AJARA ON PRORERTY MANAGEMENT AND DISPOSAL OF THE AUTONOMOUS REPUBLIC OF ADJARA

For citation: Beridze, P., (2020). The Autonomous Republic of Adjara (Based on the Example of Goderdzi Ski Resort). Globalization and Business, 10. 270-277. (In Georgian) https://doi.org/10.35945/gb.2020.10.037 


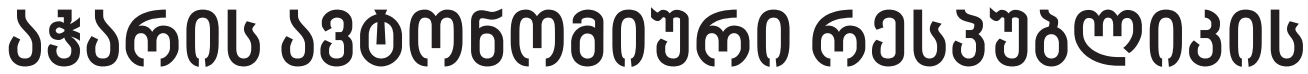

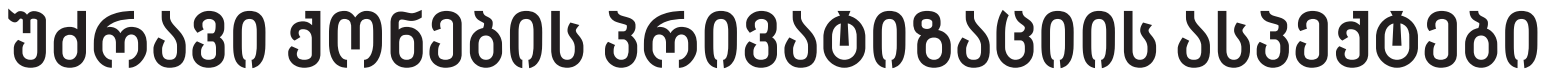

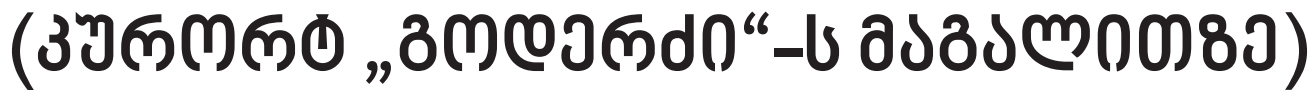

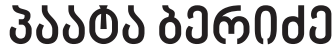

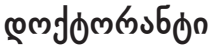

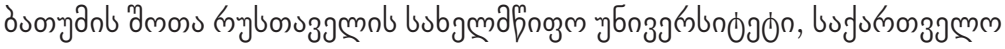 \\ paataberidze@yahoo.com
}

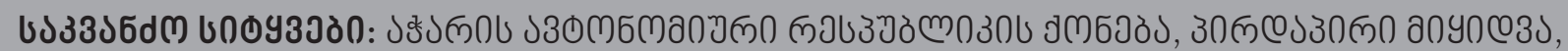

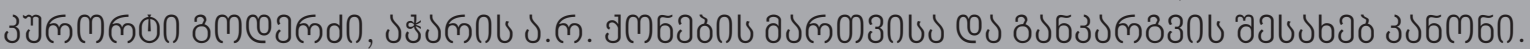

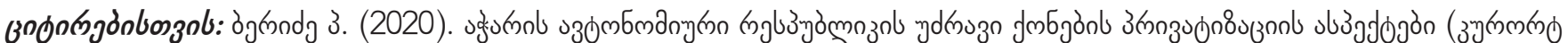

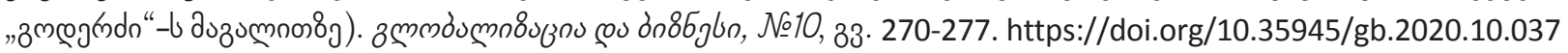

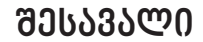

bubjgmapnogmb angk

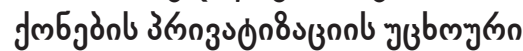

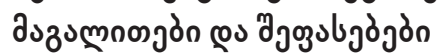

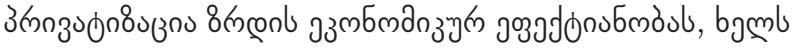

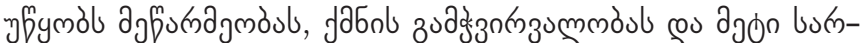

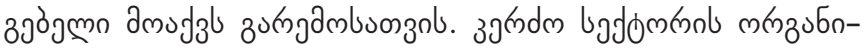

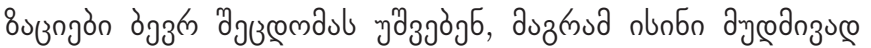

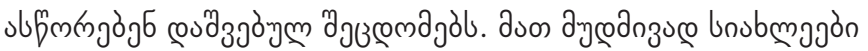

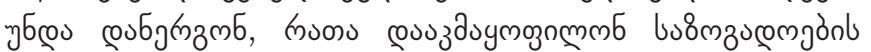

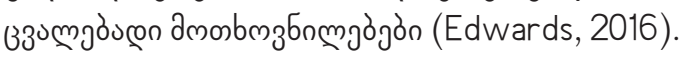

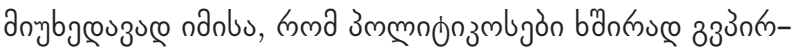

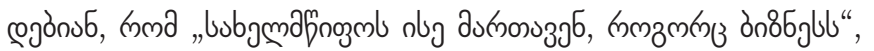

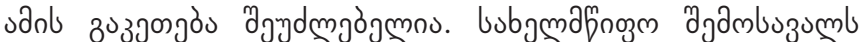

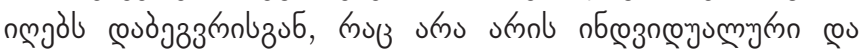

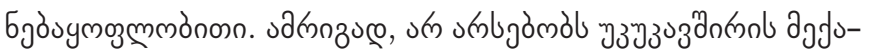

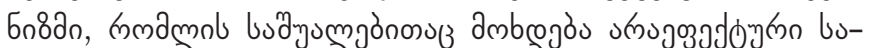

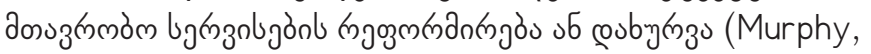
2011).

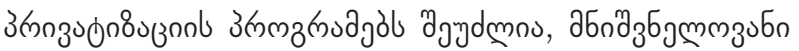

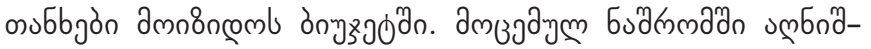

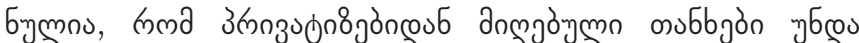

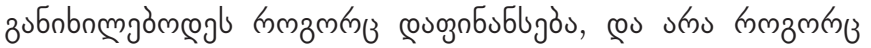

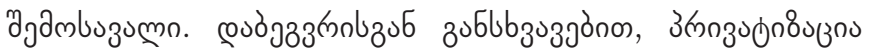

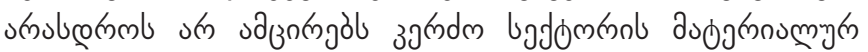
зjonnmẹsgmòul (Mackenzie, 1998).

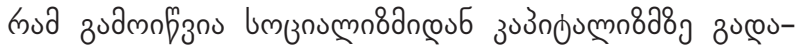

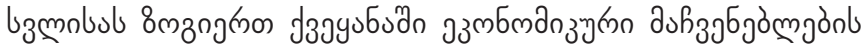

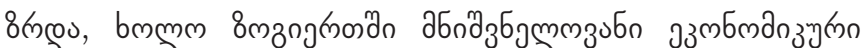

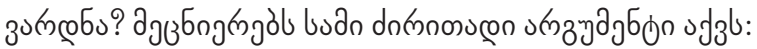

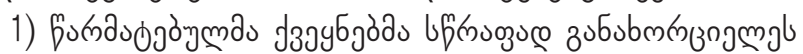

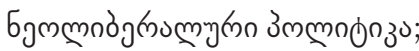

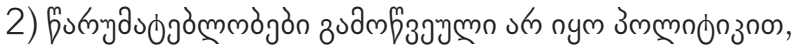

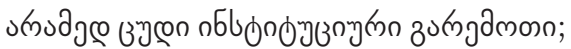

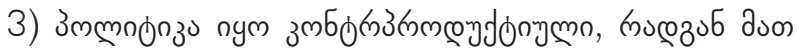

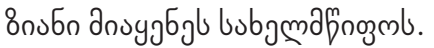

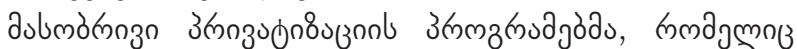

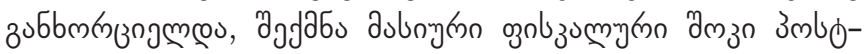

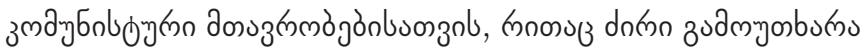

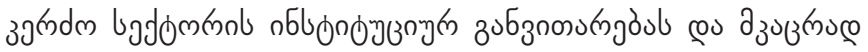

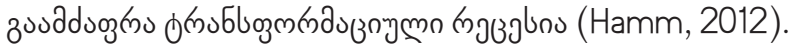

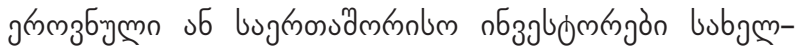

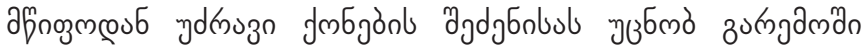

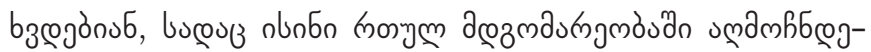

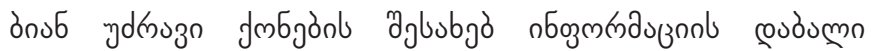

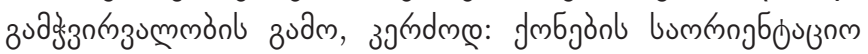

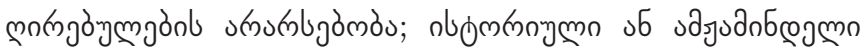

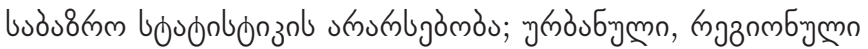

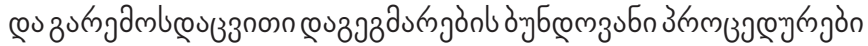

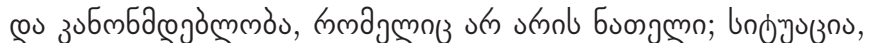

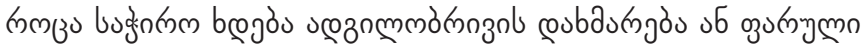

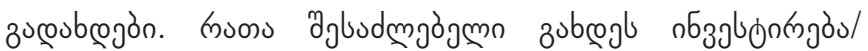

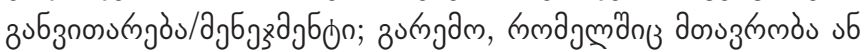

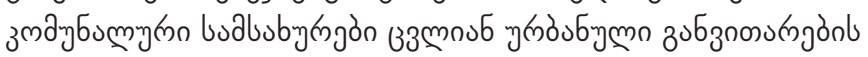




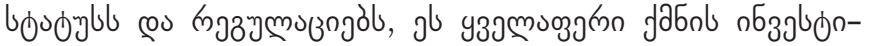

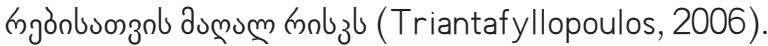

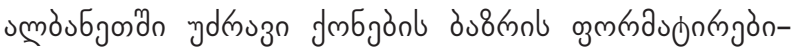

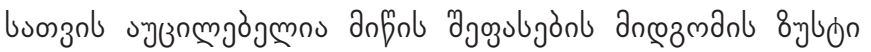

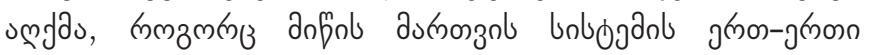

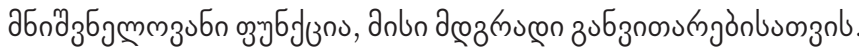

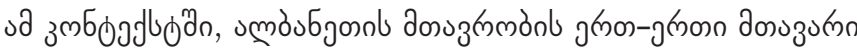

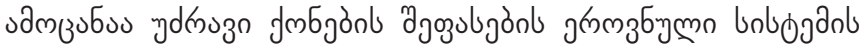

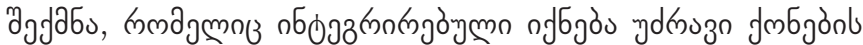
зuलubof

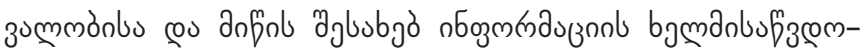

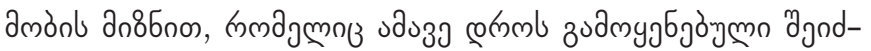
mgàu nfб

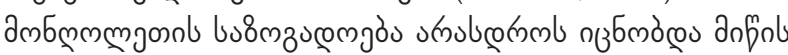

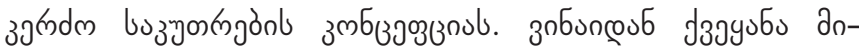

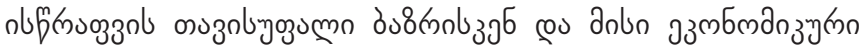

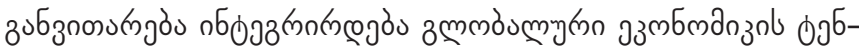

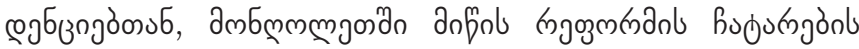

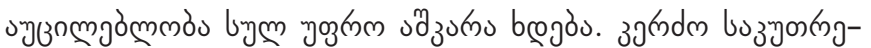

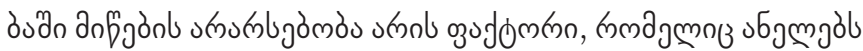

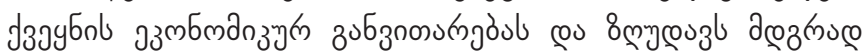

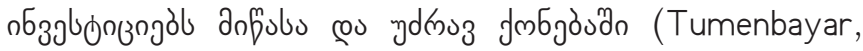
2000).

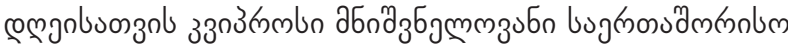

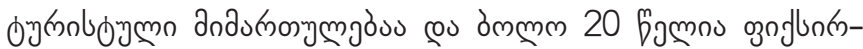

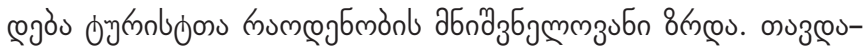

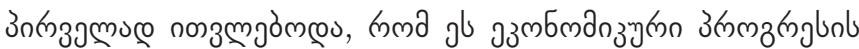

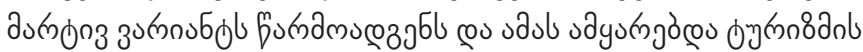

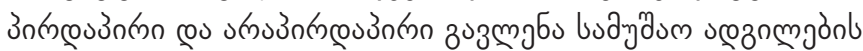

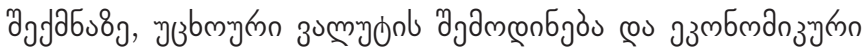

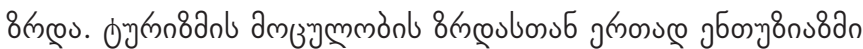

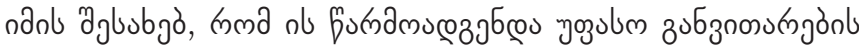

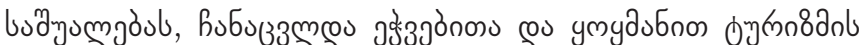

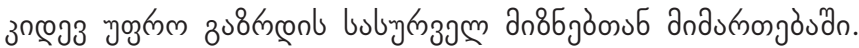

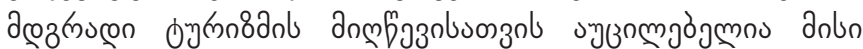

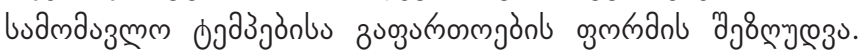

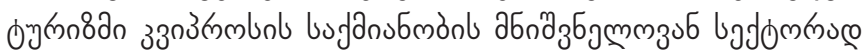

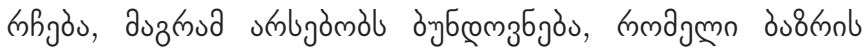
b anz n

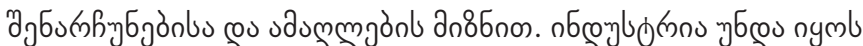

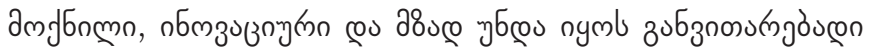

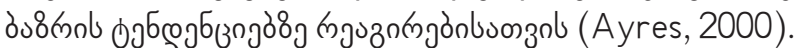

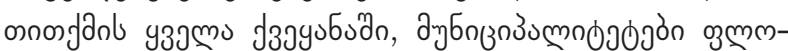

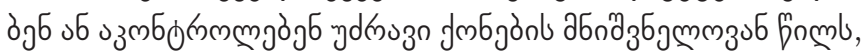

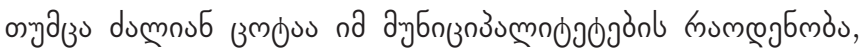

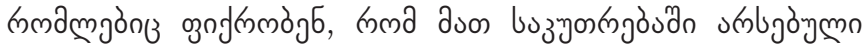

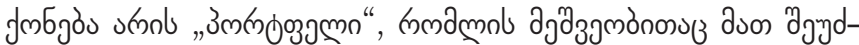

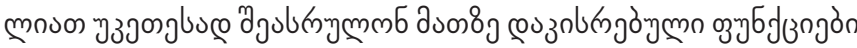
(Kaganova, 2000).

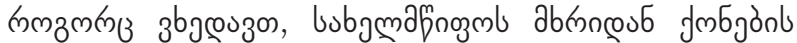

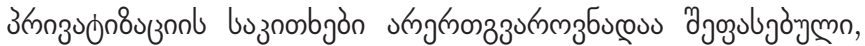

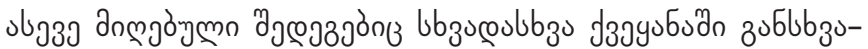

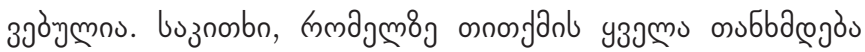

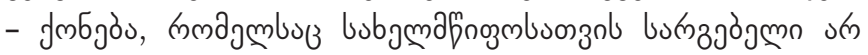

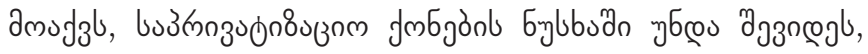

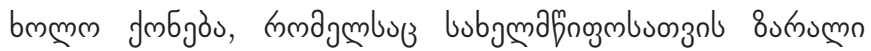

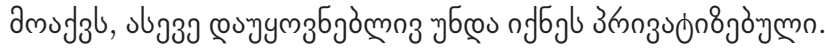

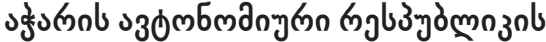

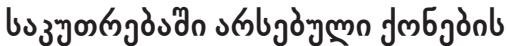

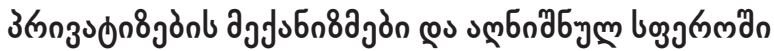

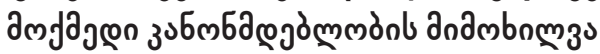

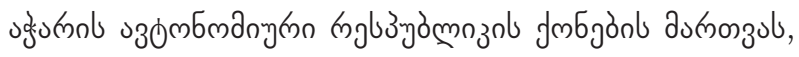

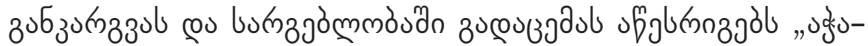

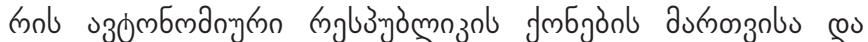

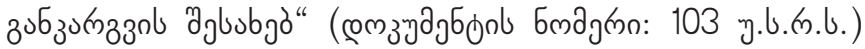

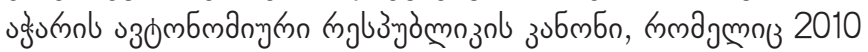

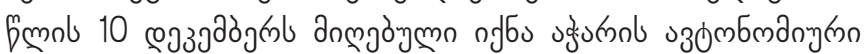

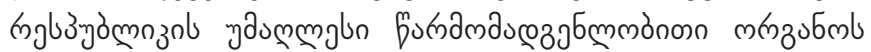

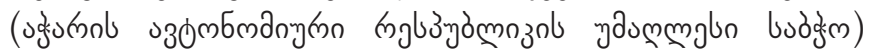

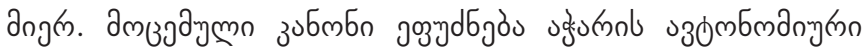

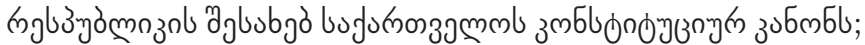

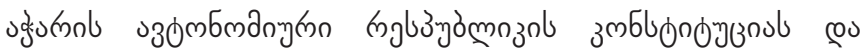

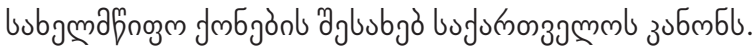

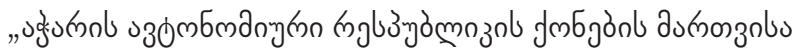

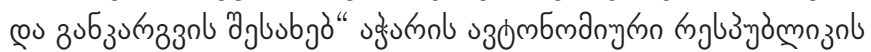

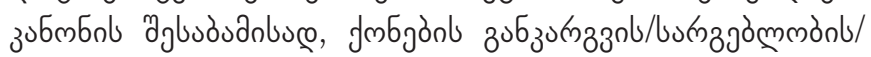

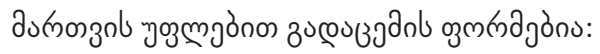

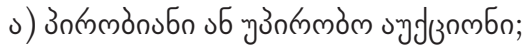

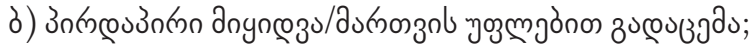

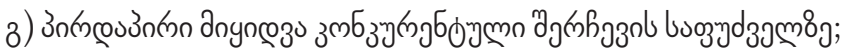

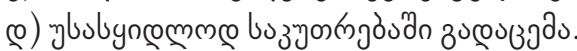

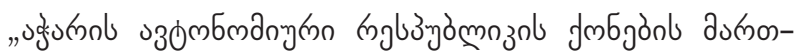

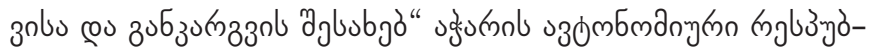

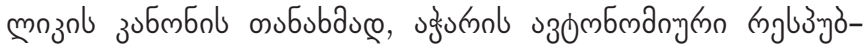

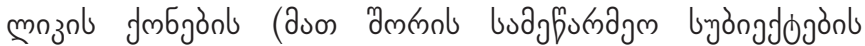

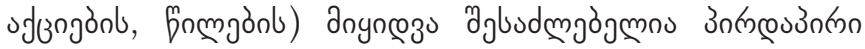

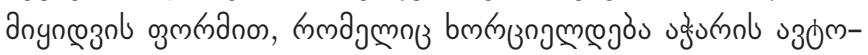

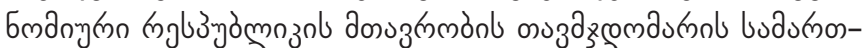

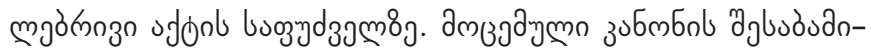

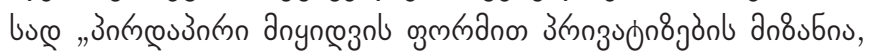

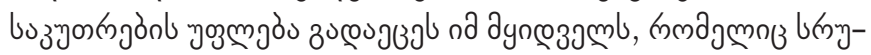

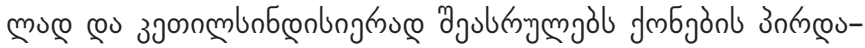

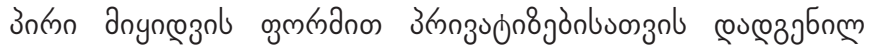

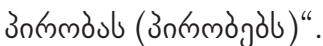




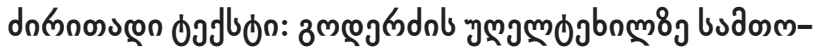

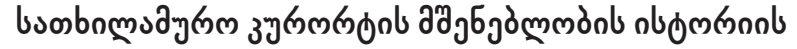

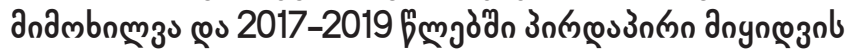

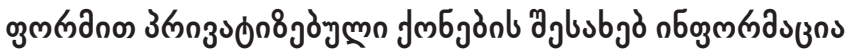

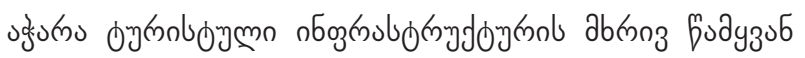
u

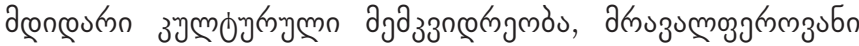

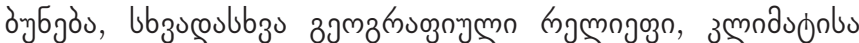

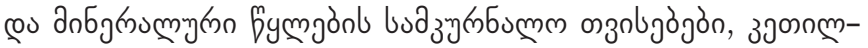

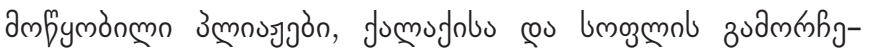

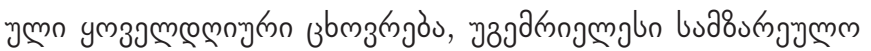

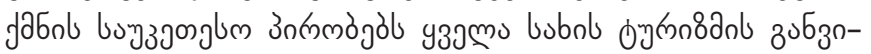

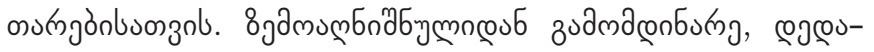

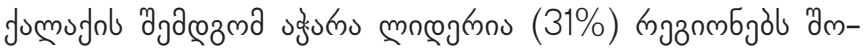

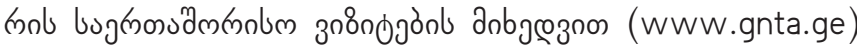
(Meskhia, 2018).

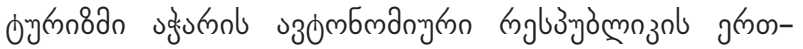

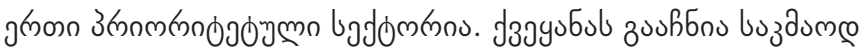

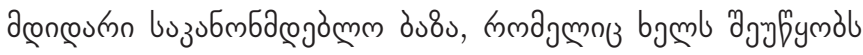

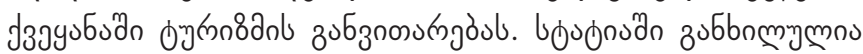

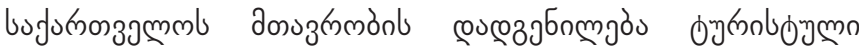

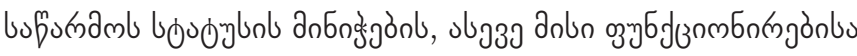

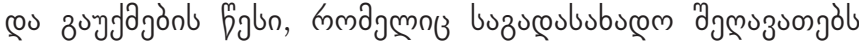

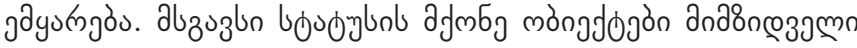

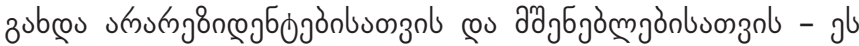

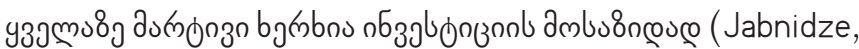
2019).

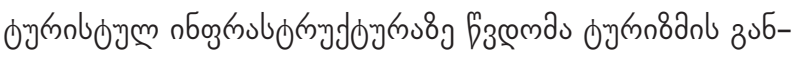

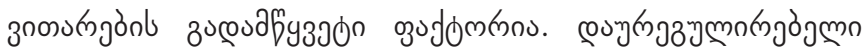

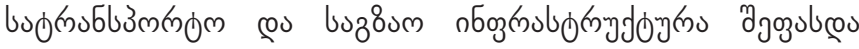

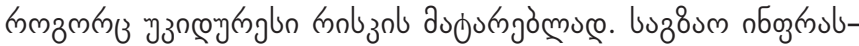

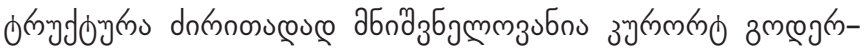

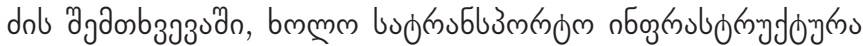

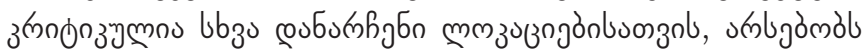

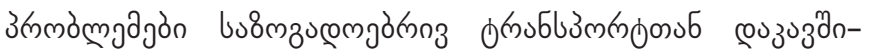

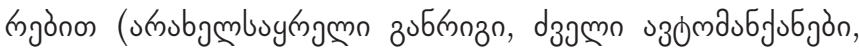

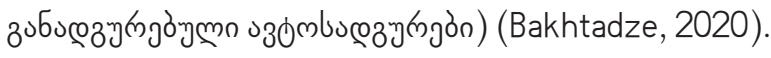

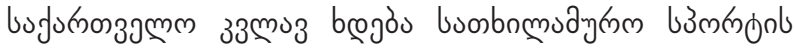

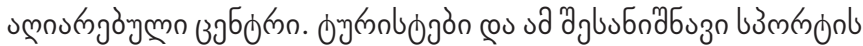

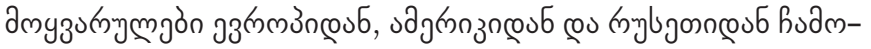

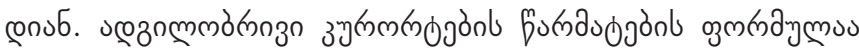

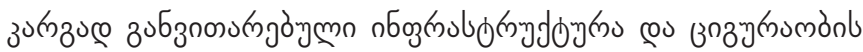

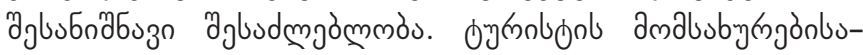

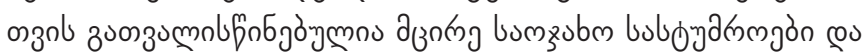

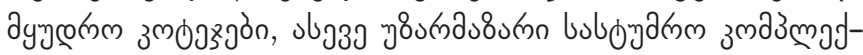
ঢgòn (Berdzenishvili, 2016).

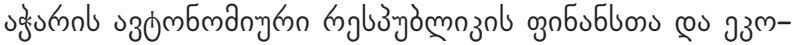
бmanzon ba

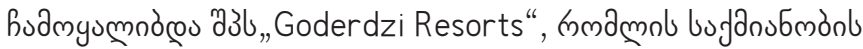

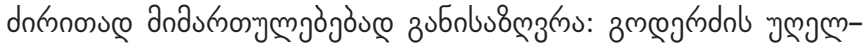

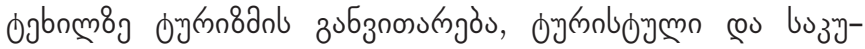

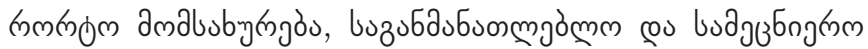

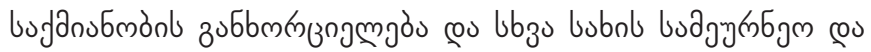

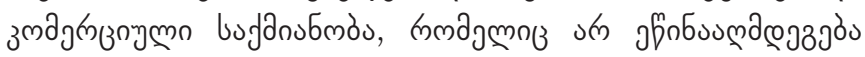

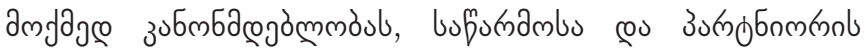

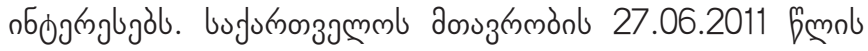

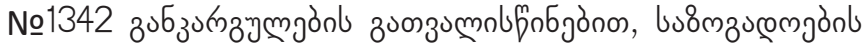

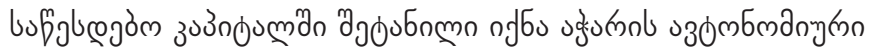

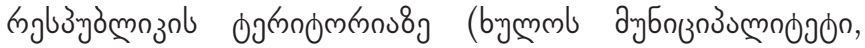

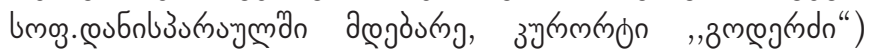

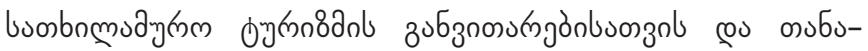

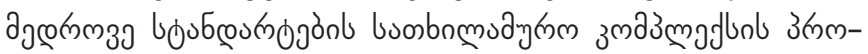
ajon

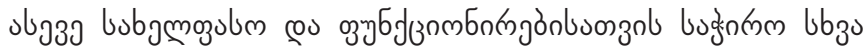

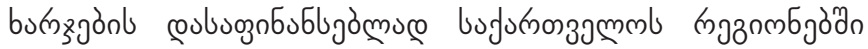

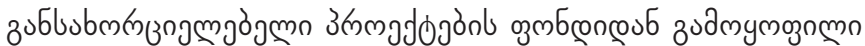
ojymuson mubbu 2000000 mutrol megbmònon, bmmm

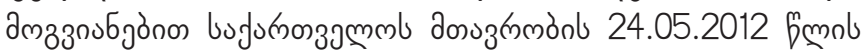
№984, 08.06.2012 Bmols №1066 (ev 13.07.2012 Bmmls

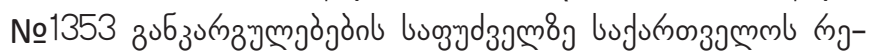

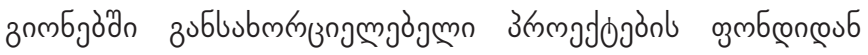

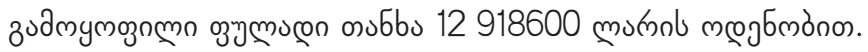

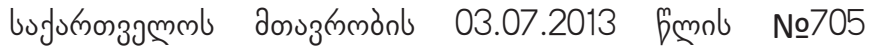

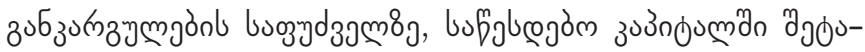

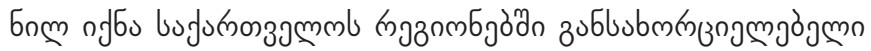

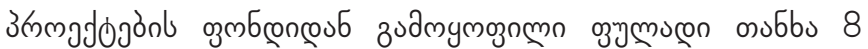

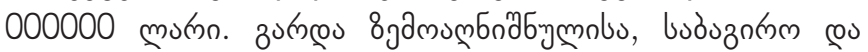

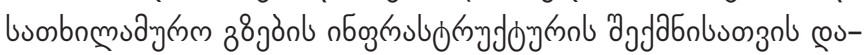
audjònon pounbumpu-23 440534 mukno.

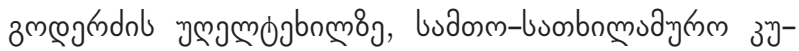

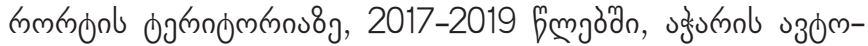

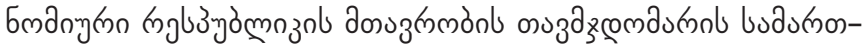

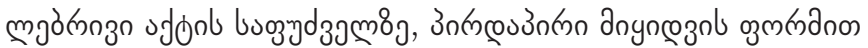

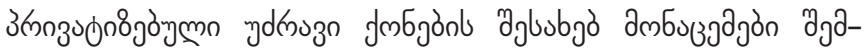

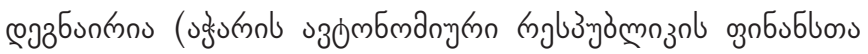

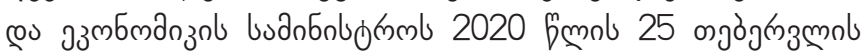
№1-01-10/879

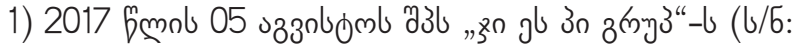

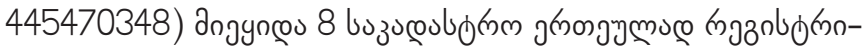

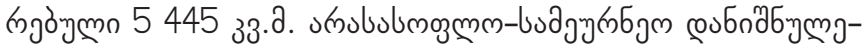

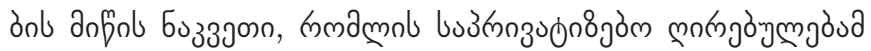

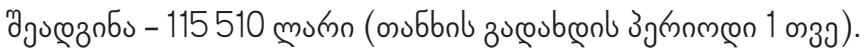

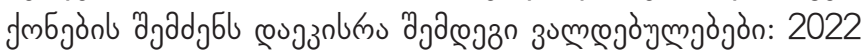

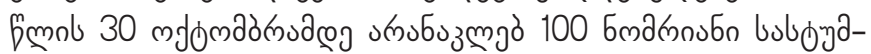

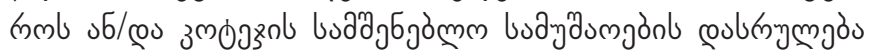

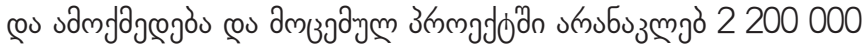
mumnl n

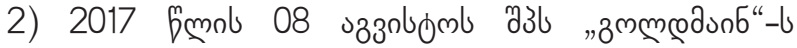

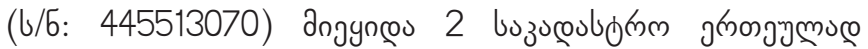




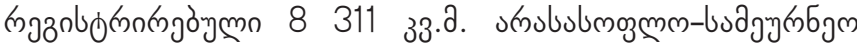

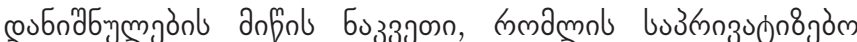

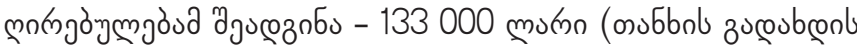

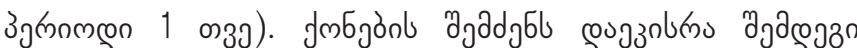

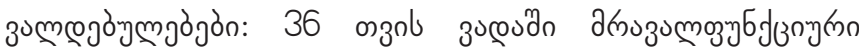

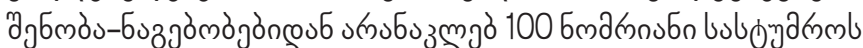

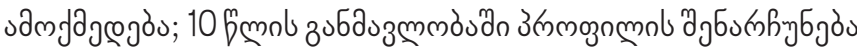

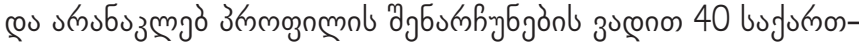

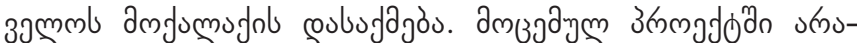

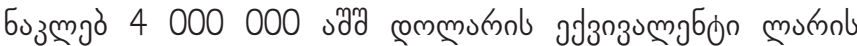

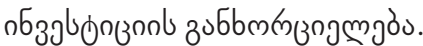

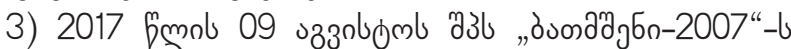
(b/6: 245442506) dngynco 2 buzucoubornm grnosgymuco

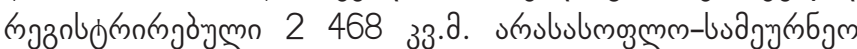

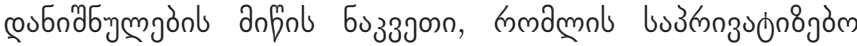

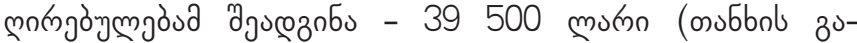

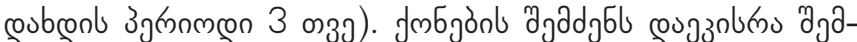

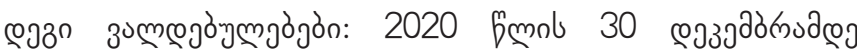

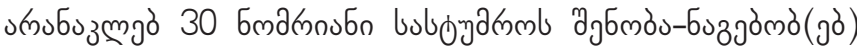

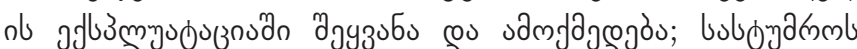

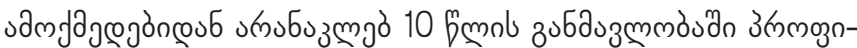

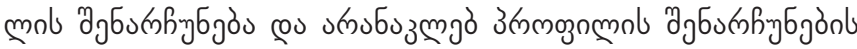

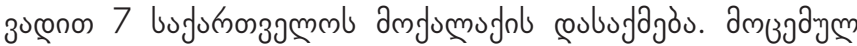

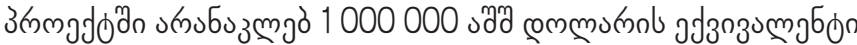

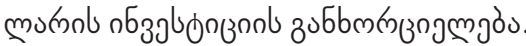

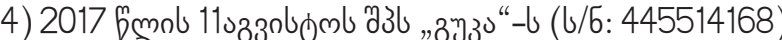

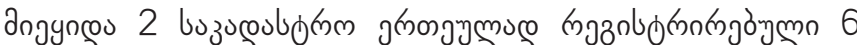

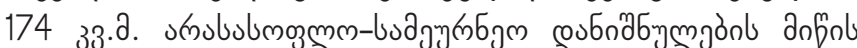

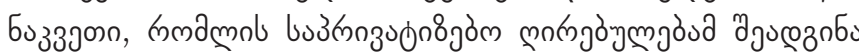

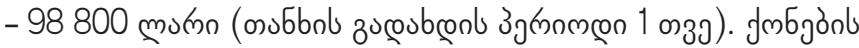

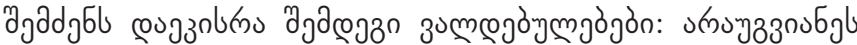

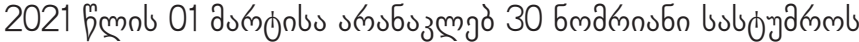

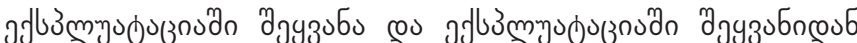

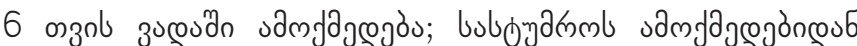

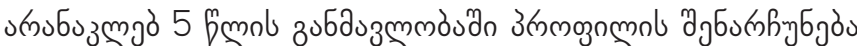

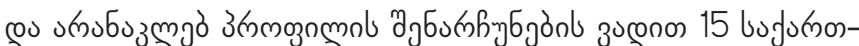

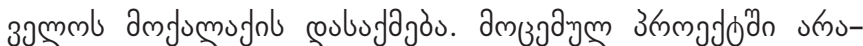

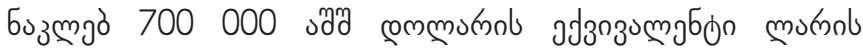

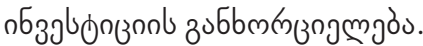

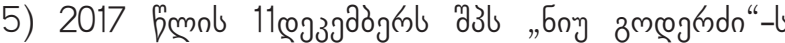
(b/б: 448052703) angyncos 1 buzucoubornm grnogymuse

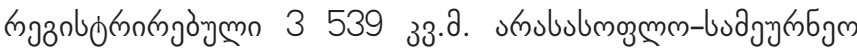

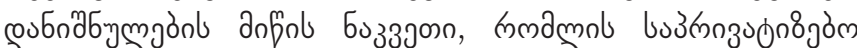

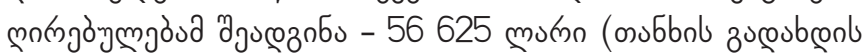

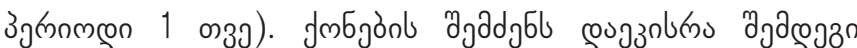

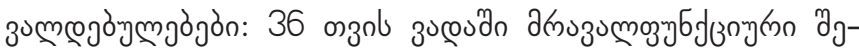

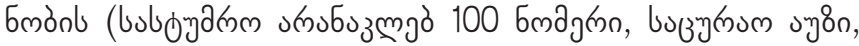

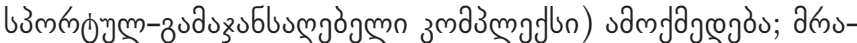

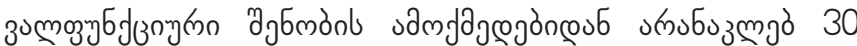

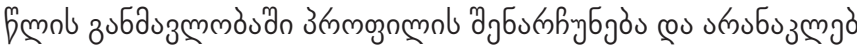

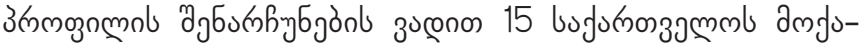

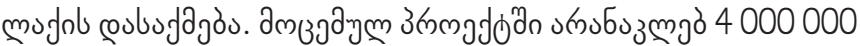
munno n

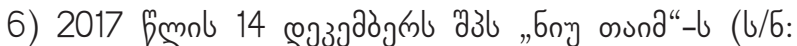

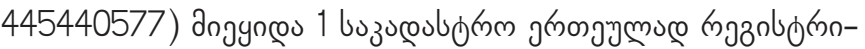

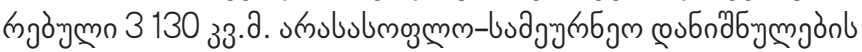

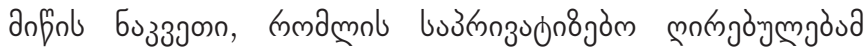
ğu@an

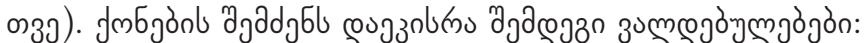

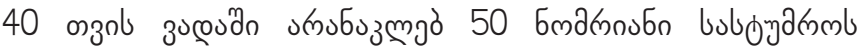

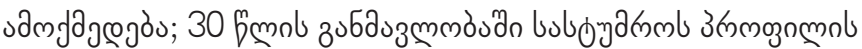

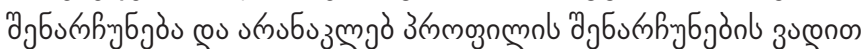

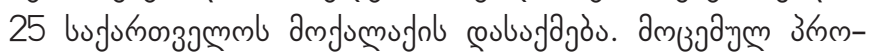

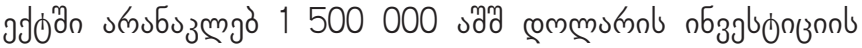

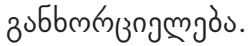

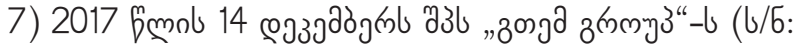

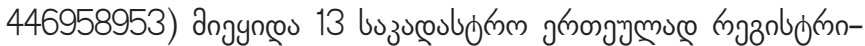

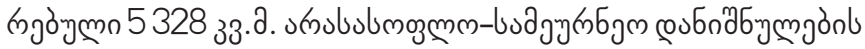

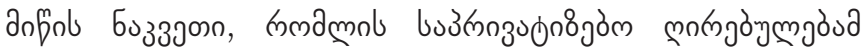

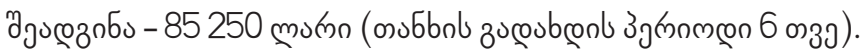

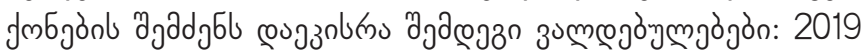

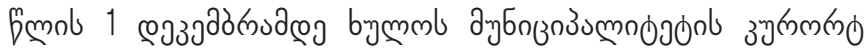

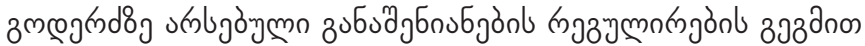

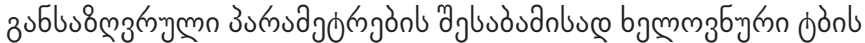

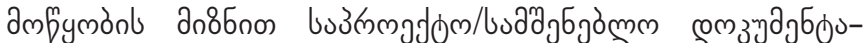

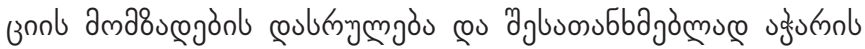

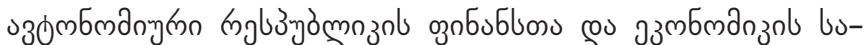

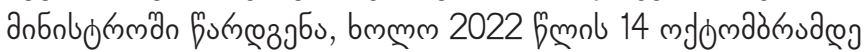
bəмmmз

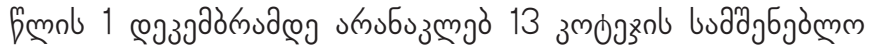

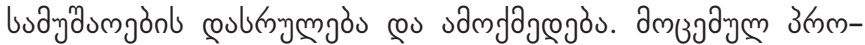

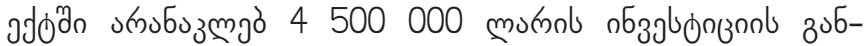

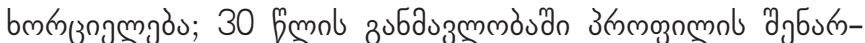

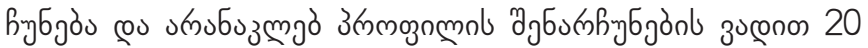
bujumonzamml amjumugno coubufajas.

8) 2018 fmols 05 nuбzuml monom nuzmöudgls (3/6:

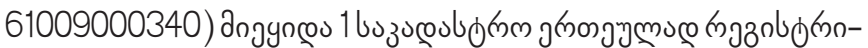

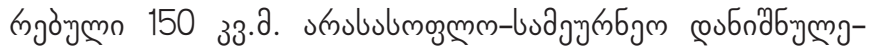

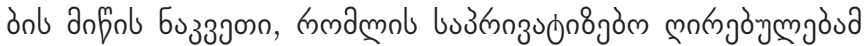

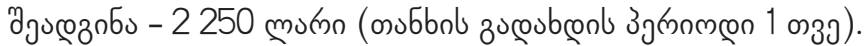

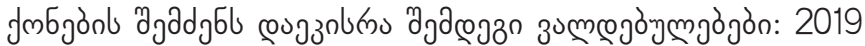

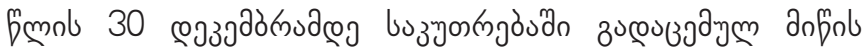

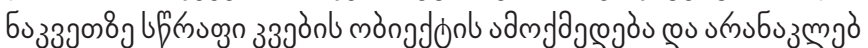
12000 mumol a

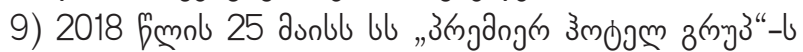

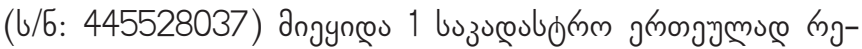

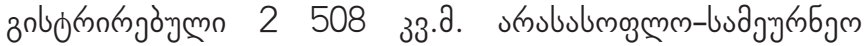

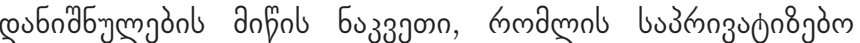

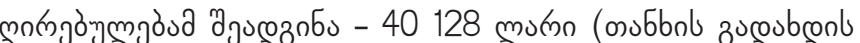

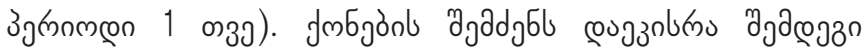




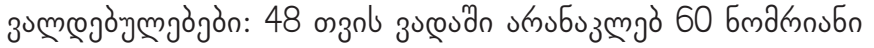

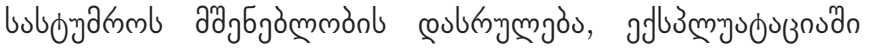

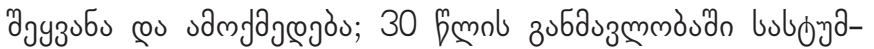

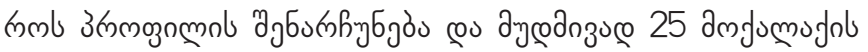

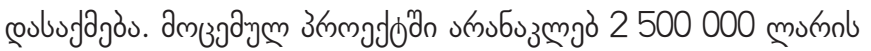

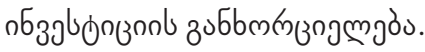

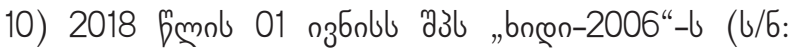

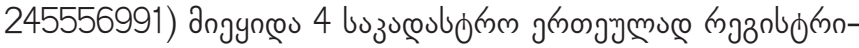

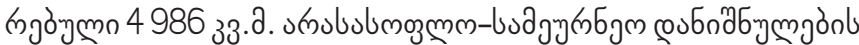

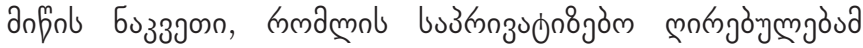

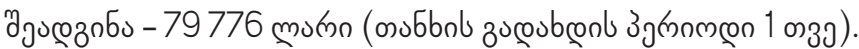

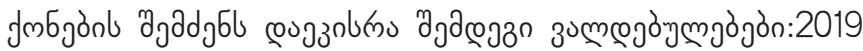

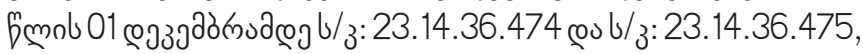

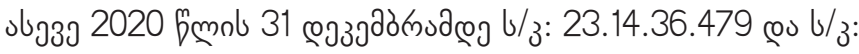

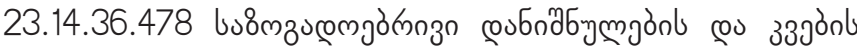

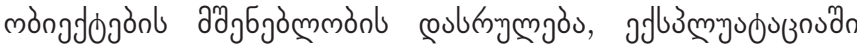

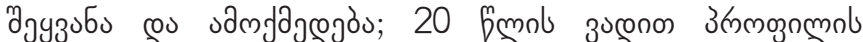

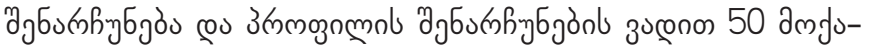

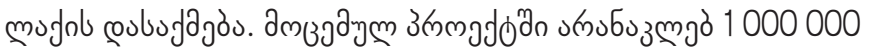
monnol nб

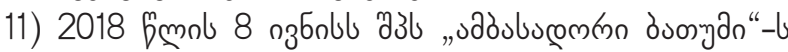
(b/6: 445515719) angyneo 1 buzusoubogn grnosgymuse

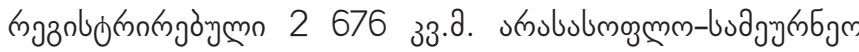

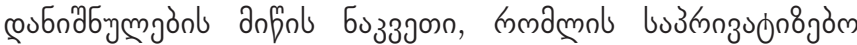

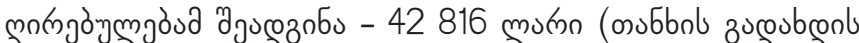

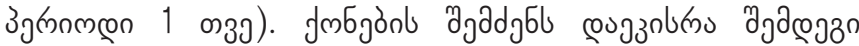

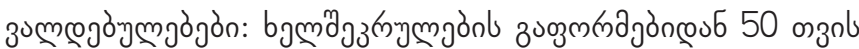

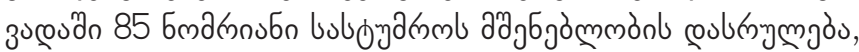

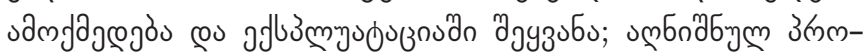

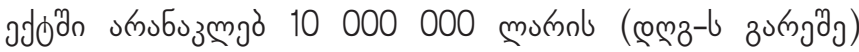

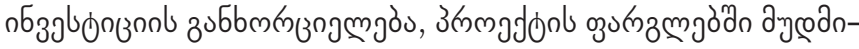

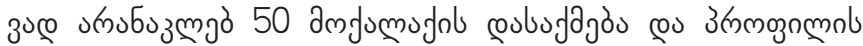

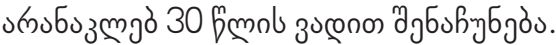

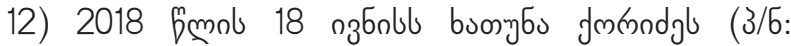

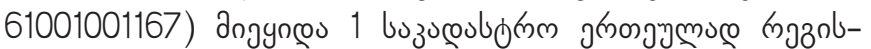

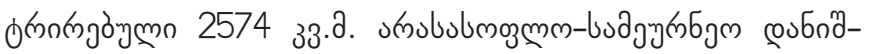

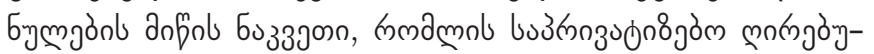

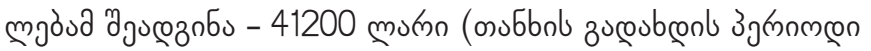

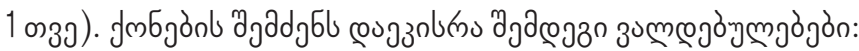

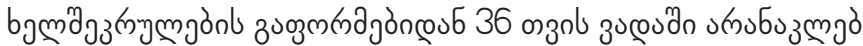

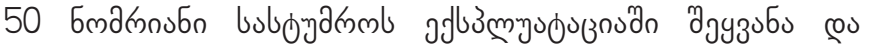

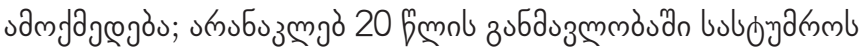

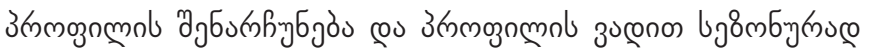

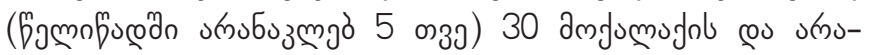

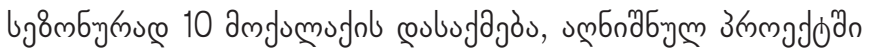

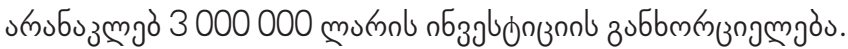

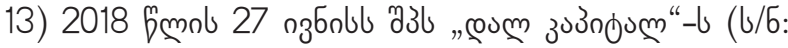

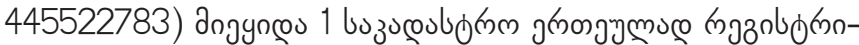

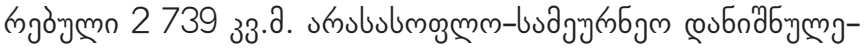

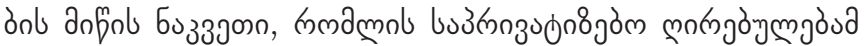

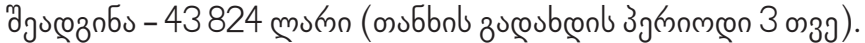

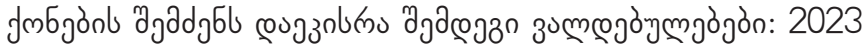

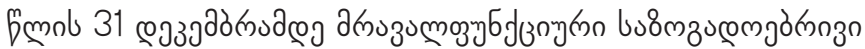

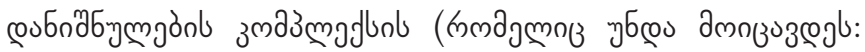

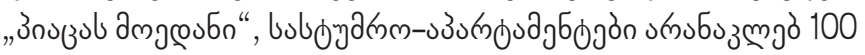

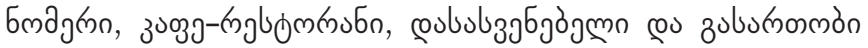

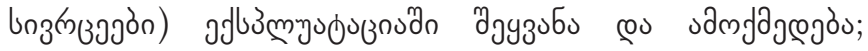

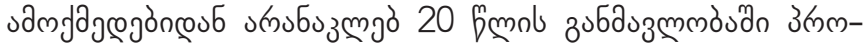

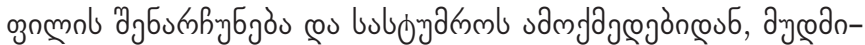

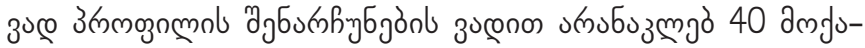

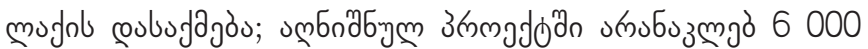

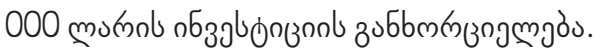

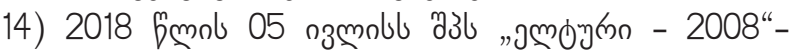

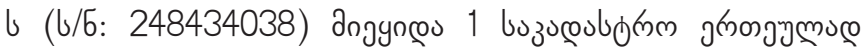

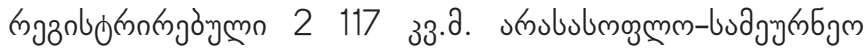

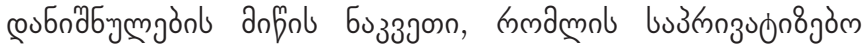

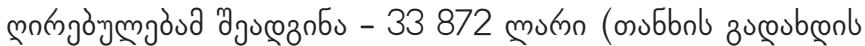

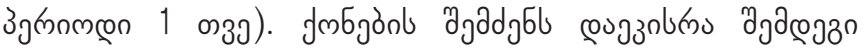

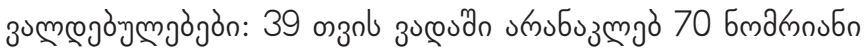

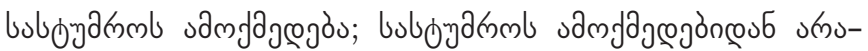

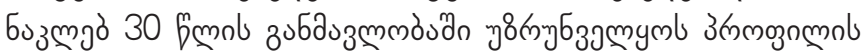

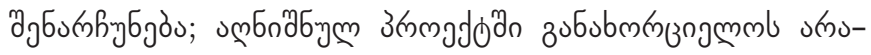

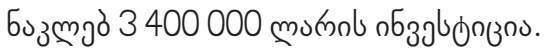

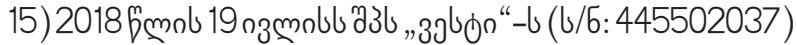

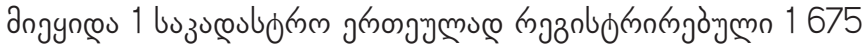

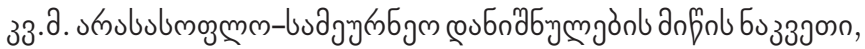

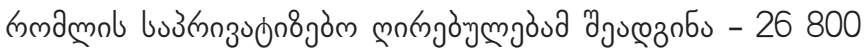

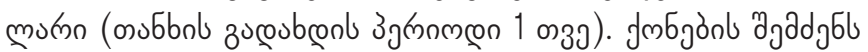

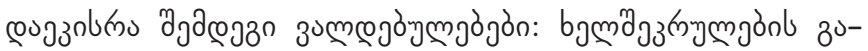

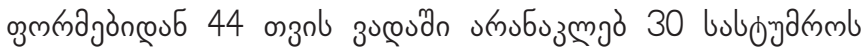

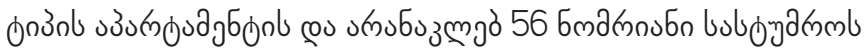

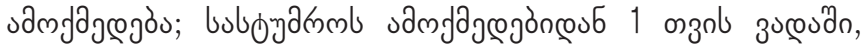

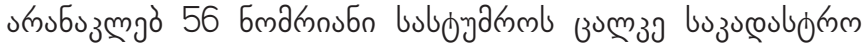

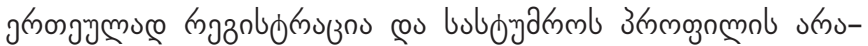

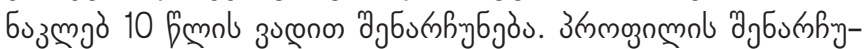

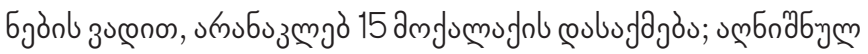

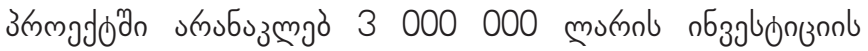
zubbmingramgojo.

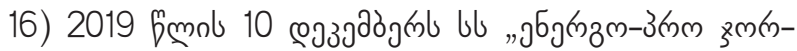

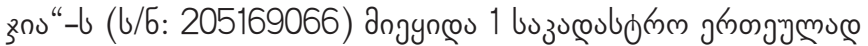

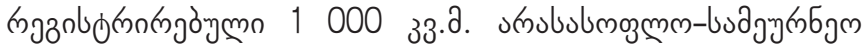

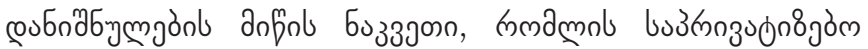

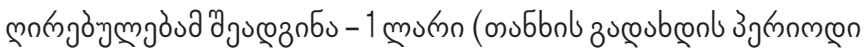

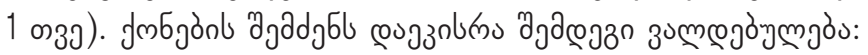

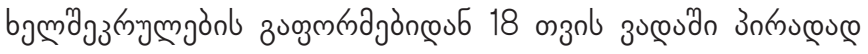

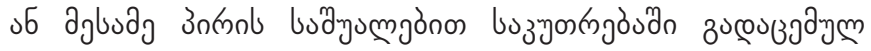

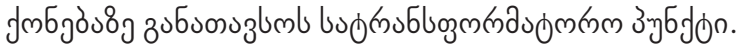

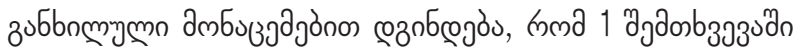

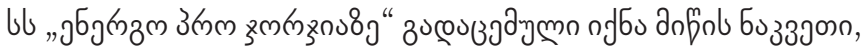

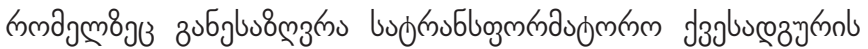




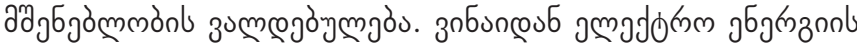

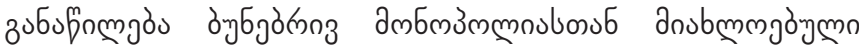

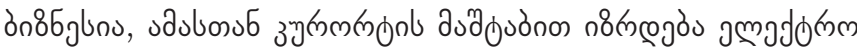

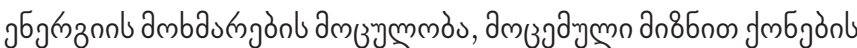

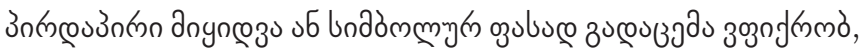

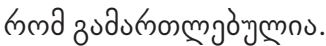

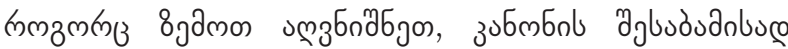

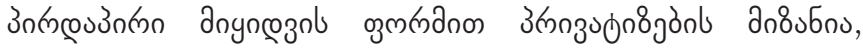

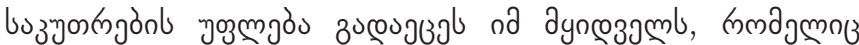

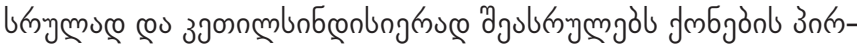

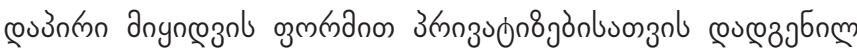

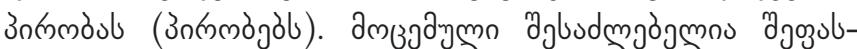

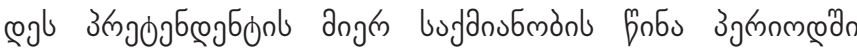

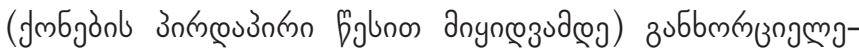

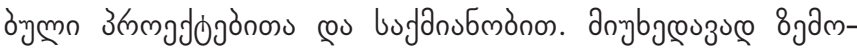

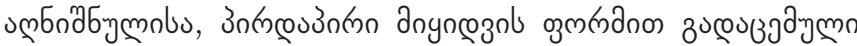

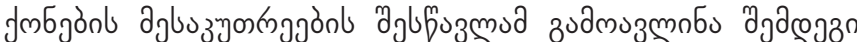
उurngamgòjòn:

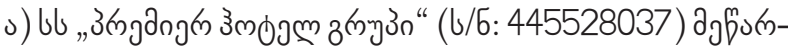

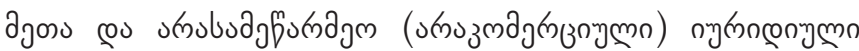
उn

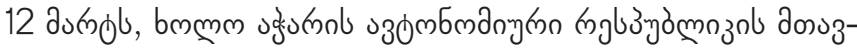

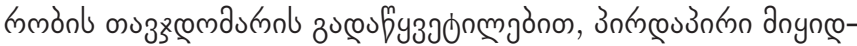

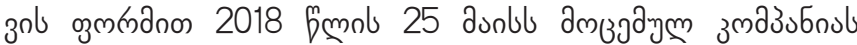

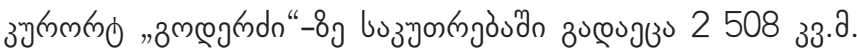
anfol bo бз390n.

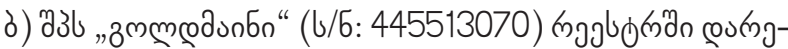

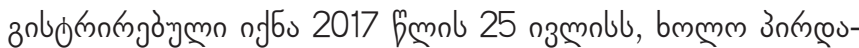

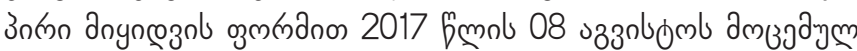

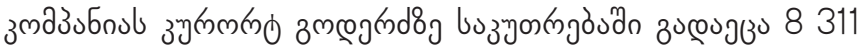

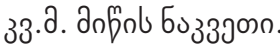

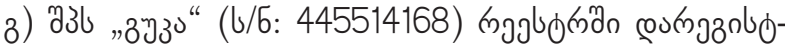

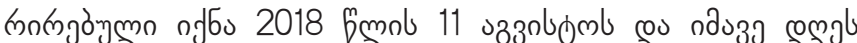

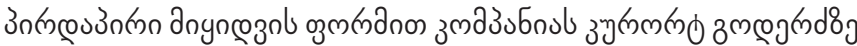

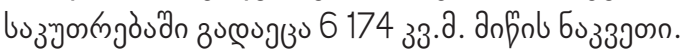

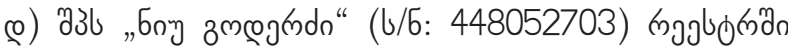

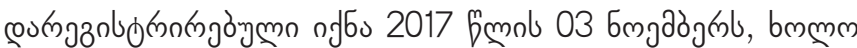

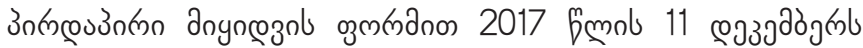

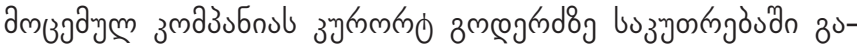

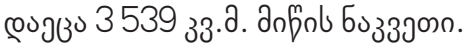

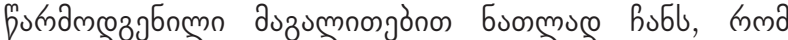

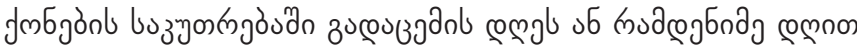

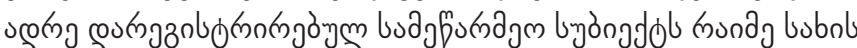

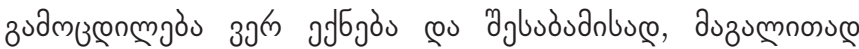

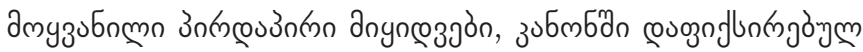

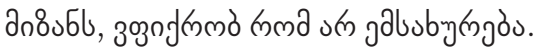

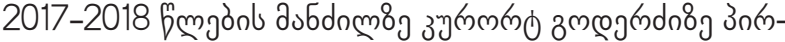

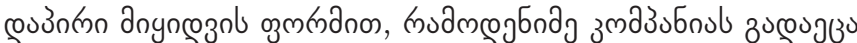

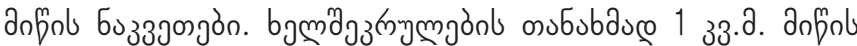

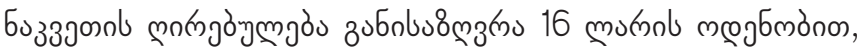

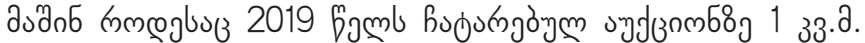

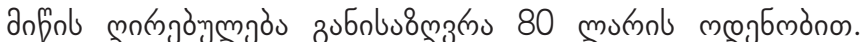

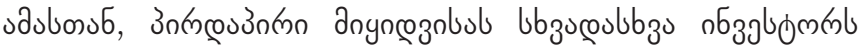

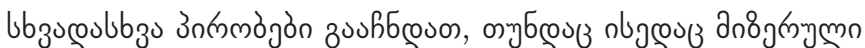

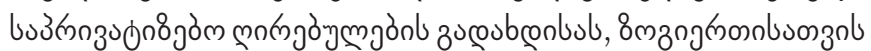

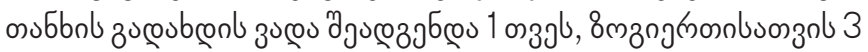

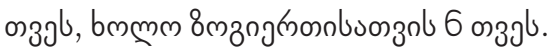

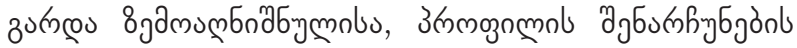

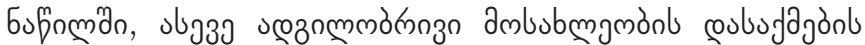

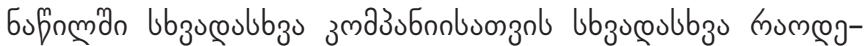

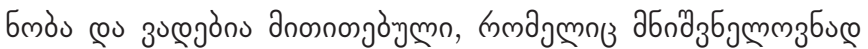

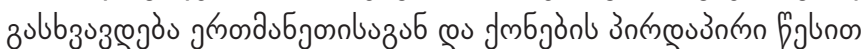

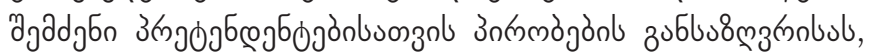

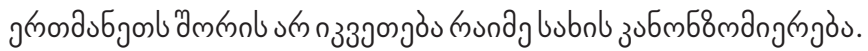

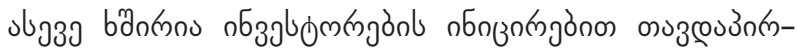

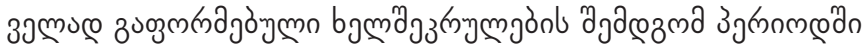

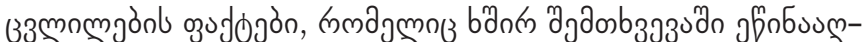

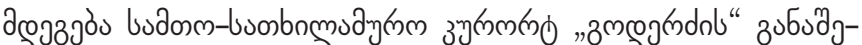

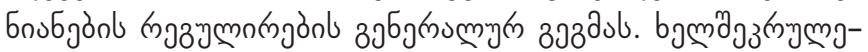

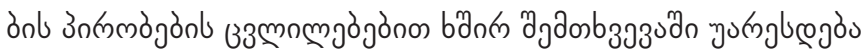

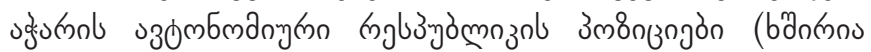

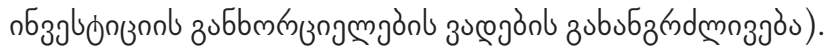

\section{cos335s}

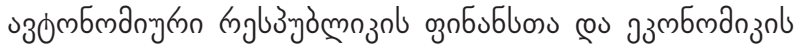

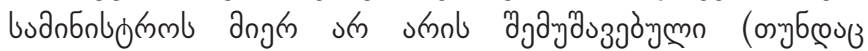

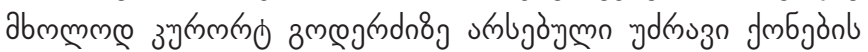

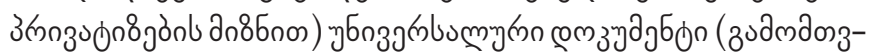

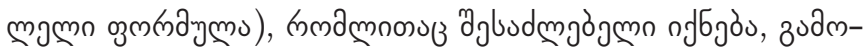

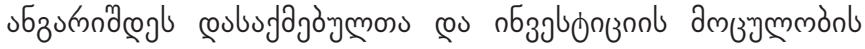

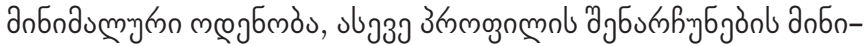

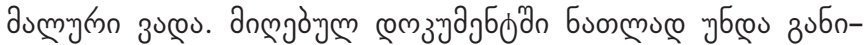

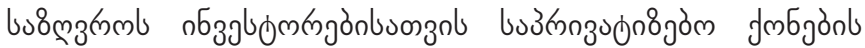

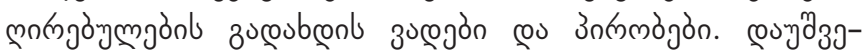

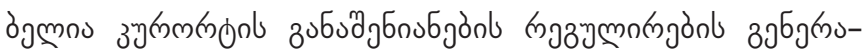

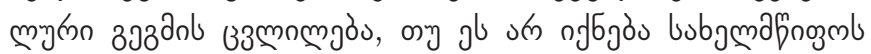

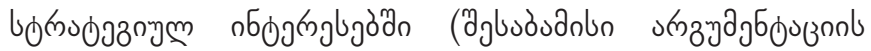

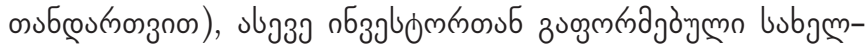

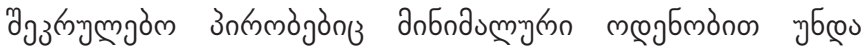
nззмjómegl.

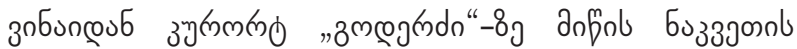

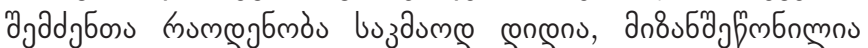

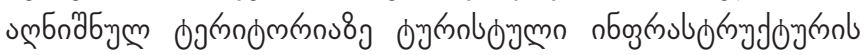

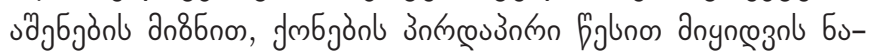

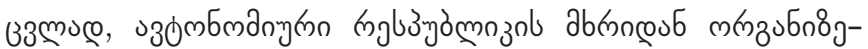

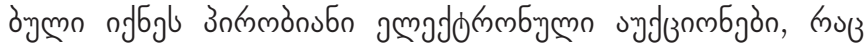

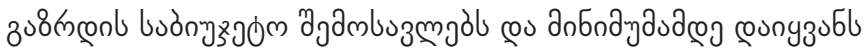

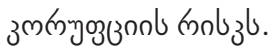




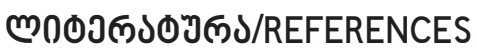

The Supreme Council of the Autonomous Republic of Ajara (2010). Law of the Autonomous Republic of Adjara on Property Management and Disposal of the Autonomous Republic of Adjara (103). (in Georgian).

Edwards, Ch., (2016). Privatization. https://www.downsizinggovernment.org/privatization

Murphy, R., P., (2011). Privatizing Federal Government Assets. The Library of Economics and Liberty. https://www.econlib. org/library/Columns/y2011/Murphyprivatization.html

Mackenzie, G. A., (1998). The Macroeconomic Impact of Privatization. IMF Economic Review https://link.springer.com/ article/10.2307/3867393.

Hamm, P., \& Lawrence P., \& King, David Stuckler (2012).Mass Privatization, State Capacity, and Economic Growth in PostCommunist Countries. American Sociological Review. Volume 77 Issue. 295-324. https://journals.sagepub.com/doi/ abs/10.1177/0003122412441354.

Kaganova, O., \& Nayyar-Stone, R., (2000). Municipal Real Property Asset Management: An Overview of World Experience, Trends and Financial Implications. Journal of Real Estate Portfolio Management Vol. 6 Issue 4.

Triantafyllopoulos, N., (2006) Constrains in Real Estate Investments in Greece 46th Congress of the European Regional Science Association: Enlargement, Southern Europe and the Mediterranean, August 30th - September 3rd, 2006, Volos, Greece https://www.econstor.eu/handle/10419/118417

Thanasi, Marsela., (2014). Property Mass Evaluation in Albania. Journal of Marketing \& Management. Nov 2014, Vol. 5 2, 1-11. 11. https://web.a.ebscohost.com/abstract?direct=true\&profile=ehost\&scope=site\&authtype=crawler\&jrnl=21539715\& AN=96153032\&h=woVh117EyZeuZnq9MPiqHoc3ArL\%2fWh2FIKpZcF4qfkFIdwlxuOWpYbg\%2bDvftCe0KaaNVXpIW5UBbi 010\%2fscOSw\%3d\%3d\&crl=c\&resultNs=AdminWebAuth\&resultLocal=ErrCrINotAuth\&crlhashurl=login.aspx\%3fdirect\% 3dtrue\%26profile\%3dehost\%26scope\%3dsite\%26authtype\%3dcrawler\%26jrnl\%3d21539715\%26AN\%3d96153032

Tumenbayar, Nyamaa., (2000). Land Privatization Option for Mongolia 2000 Constituting the Commons: Crafting Sustainable Commons in the New Millennium, the Eighth Biennial Conference of the International Association for the Study of Common Property. http://dlc.dlib.indiana.edu/dlc/handle/10535/133

Ron, Ayres., (2000). Tourism as a passport to development in small states: reflections on Cyprus International Journal of Social Economics 1 February 2000 https://www.emerald.com/insight/content/doi/10.1108/03068290010308992/full/html

Bakhtadze, E., Phalavandishvili, N., \& Robitashvili, N., (2020). IDENTIFYING TOURISM MARKET GROWTH OPPORTUNITIES AND RISKS IN THE AUTONOMOUS REPUBLIC IN AJARA (GEORGIA). Proceedings of the 2020 International Conference «ECONOMIC SCIENCE FOR RURAL DEVELOPMENT» No 54 Jelgava, LLU ESAF, 12-15 May 2020, pp. 35-42 DOI: 10.22616/ ESRD.2020.54.004. http://www.esaf.Ilu.Iv/sites/esaf/files/files/lapas/Krajums_Nr_54_07.07.2020.pdf\#page=35

Meskhia, E., (2018). The Role of Adjara Sightseeing in Tourism Proceedings of the International scientific and practical conference - Bulgaria of regions.

Berdzenishvili, N., (2016). The Mountain Resorts of Georgia Published in the Russian Federation Tourism Education Studies and Practice Has been issued since 2014. ISSN: 2312-0037 E-ISSN: 2409-2436 7, 1, 4-9, 2016 http://ejournal10.com/ journals_n/1463828479.pdf

Jabnidze, N., Gechbaia, B., Phalavandishvili, N., \& Bakhtadze, E., (2019). An enterprise with tourist status as a stimulant for attracting investments and tourists to Adjara Fundamental and applied researches in practice of leading scientific schools, 31(1),77-80 Accepted 28.02.2019 https://farplss.org/index.php/journal/article/view/571/531 